J. Nonlinear Var. Anal. 4 (2020), No. 1, pp. 135-151

Available online at http://jnva.biemdas.com

https://doi.org/10.23952/jnva.4.2020.1.10

\title{
ON THE EXISTENCE AND APPROXIMATION OF SOLUTIONS FOR BILEVEL VECTOR VARIATIONAL INEQUALITIES IN BANACH SPACES
}

\author{
O. CHADLI ${ }^{1, *}$, R. N. MOHAPATRA ${ }^{2}$ \\ ${ }^{1}$ Department of Economics, Ibn Zohr University, Agadir, Morocco \\ ${ }^{2}$ Department of Mathematics, University of Central Florida, Orlando, USA
}

\begin{abstract}
In this paper, a bilevel vector variational inequality problem is investigated in the setting of Banach spaces. We propose a method which consists in reducing the study of the bilevel vector variational inequality problem to that of a one level vector problem. By introducing a vector auxiliary principle, we propose an algorithm to approximate the solution of the considered bilevel vector variational inequality problem. The strong convergence of the proposed iterative method is studied.
\end{abstract}

Keywords. Bilevel vector variational inequalities; $C$-monotone operators; $C$-pseudomonotone operators; Auxiliary principle; Convergence analysis.

\section{INTRODUCTION}

The theory of vector variational inequalities began with the pioneering work of Giannessi [6] in 1980. He extended the classical variational inequality for vector-valued functions in the setting of finite dimensional spaces. Since then, a large number of papers have appeared in the literature on different aspects of vector variational inequalities. Later, it was shown that the theory of vector variational inequalities is a powerful tool to study vector optimization problems.

Bilevel vector inequality problem is defined as a mathematical program, where an upper level vector variational inequality problem contains another vector variational inequality problem as a constraint, called the lower level problem. This problem is motivated by the work of many researchers which we mention briefly. Harker and Pang [7] in 1988 considered the bilevel optimization problem, in which the constraint set is the solution set of a variational inequality or complementarity problem. Afterwards, a large number of papers on this topic have appeared in the literature, see, for instance, $[1,4,14,15]$ and the references therein. Bonnel and Morgan [2] considered a bilevel optimization problem with a vector-valued objective function on the lower level and a scalar-valued objective function on the upper level. They called it a semivectorial bilevel optimization problem. Liou, Yang and Yao [12] also studied such problems and called them mathematical programs with vector optimization. Recently, Ju, Zhu and Akram [9] studied Levitin-Polyak well posedness for bilevel vector variational inequalities. For applications, many real life problems can be modeled as a bilevel problem. Such models appear for instance

\footnotetext{
${ }^{*}$ Corresponding author.

E-mail addresses: o.chadli@uiz.ac.ma (O.Chadli),ram.mohapatra@ucf.edu (R.N. Mohapatra).

Received December 27, 2019; Accepted March 25, 2020.
}

(C)2020 Journal of Nonlinear and Variational Analysis 
in the study of transportation (network design, optimal pricing) problems, economics (Stackelberg games, principal-agent problem, taxation, policy decisions), management (network facility location, coordination of multi-divisional firms), engineering (optimal design); see [3].

In this paper, we study the existence and approximation of solutions for bilevel vector variational inequalities in the setting of Banach spaces. Our approach consists of reducing the study of the bilevel vector variational inequality problem to that of a one level vector problem. By considering a vector auxiliary principle, we generate an iterative sequence. This leads us to propose an algorithm to approximate the solution of the considered bilevel vector variational inequality problem. Afterward, we study the strong convergence of the sequence generated by the algorithm to the solution of the bilevel vector variational inequality problem considered in this paper.

The paper is organized as the following. In Section 2, we give a description of the problem studied in this paper as well as some definitions and preliminary results. In Section 3, we study the existence of solutions of the bilevel problem considered by a method which consists to reduce the study of the bilevel vector variational inequality problem to that of a one level vector problem. First, we study the existence of solutions of the considered one level vector variational inequality problem and then we use this approach to derive some existence results for the bilevel vector variational inequality problem considered in this paper. Section 4 is devoted to the approximation of solutions for the bilevel vector variational inequality problem. By introducing a vector auxiliary principle, we propose an algorithm and study the strong convergence of the generated sequence to a solution of the bilevel problem considered.

\section{PROBLEM FORMULATION AND PRELIMINARIES}

Let $X$ and $Z$ be two Banach spaces, and let $K$ be a nonempty, closed and convex subset of $X$. Let $C \subset Z$ be a proper convex cone such that its topological interior, denoted by $\operatorname{int}(C)$, is nonempty. For $M \subseteq X$, we denote by $\operatorname{conv}(M)$ the convex hull of $M$ and by $\operatorname{cl}(M)$ the closure of $M$. We denote by $Z^{*}$ the dual space of $Z$ and by $C^{*}$ the positive polar cone of $C$, i.e.,

$$
C^{*}=\left\{\lambda \in Z^{*}:\langle\lambda, z\rangle \geq 0 \text { for all } z \in C\right\} .
$$

Note that

$$
\operatorname{int}\left(C^{*}\right) \subset\left\{\lambda \in Z^{*}:\langle\lambda, z\rangle>0 \text { for all } z \in \operatorname{int}(C)\right\} .
$$

Further, we denote by $\mathscr{L}(X, Z)$ the space of all bounded linear operators from $X$ into $Z$, and by $\langle l, x\rangle$ the value of $l \in \mathscr{L}(X, Z)$ at $x \in X$. For $\varpi \in \operatorname{int}\left(C^{*}\right)$ and $T: X \rightarrow \mathscr{L}(X, Z)$, we define the operator $T_{\varpi}: X \rightarrow X^{*}$ by $\left\langle T_{\varpi}(x), y\right\rangle=\langle\varpi,\langle T(x), y\rangle\rangle$, for all $x, y \in X$.

Let $F: K \rightarrow \mathscr{L}(X, Z)$ be two operators and $\varphi: K \rightarrow Z$ be a vector-valued function. We consider the following mixed vector variational inequality: Find $\bar{x} \in K$ such that

$$
\langle F(\bar{x}), y-\bar{x}\rangle+\varphi(y)-\varphi(\bar{x}) \notin-\operatorname{int}(C) \text {, for all } y \in K \text {. }
$$

Our attention will be focused on the possibility of computing solutions of (2.1) with respect to a given operators $G: K \rightarrow \mathscr{L}(X, Z)$. More precisely, we are interested in solving the following problem: Find $x^{*} \in \mathbb{S}$ such that

$$
\left\langle G\left(x^{*}\right), y-x^{*}\right\rangle \notin-\operatorname{int}(C) \text {, for all } y \in \mathbb{S},
$$

where the set of constraints $\mathbb{S}$ is the solution set of (2.1). That is,

$$
\mathbb{S}=\{x \in K:\langle F(x), y-x\rangle+\varphi(y)-\varphi(x) \notin-\operatorname{int}(C) \text {, for all } y \in K\} .
$$


Problem (2.2) is referred to bilevel vector variational inequality, or of hierarchical type problem in the literature, because the set of constraints $\mathbb{S}$ is it self given as subproblem.

We shall need the following lemma for the solution of our problem.

Lemma 2.1. For $x, y \in C$, we have

$$
x \in C \text { and } y \notin-\operatorname{int}(C) \quad \text { implies } \quad x+y \notin-\operatorname{int}(C) .
$$

Below, we give some definitions and preliminary notions that we will need in the sequel.

Definition 2.1. A vector-valued function $\varphi: K \rightarrow Z$ is said to be $C$-convex if, for each $x, y \in K$ and $t \in[0,1]$,

$$
t \varphi(x)+(1-t) \varphi(y)-\varphi(t x+(1-t) y) \in C .
$$

The function $\varphi$ is said to be $C$-concave if $-\varphi$ is $C$-convex.

Definition 2.2. The operator $F: K \rightarrow \mathscr{L}(X, Z)$ is said to be

(a) $C$-monotone if $\langle F(x)-F(y), x-y\rangle \in C$ for all $x, y \in K$;

(b) $C$-upper hemicontinuous if, for all $x, y, z \in K$, the vector-valued function $t \in[0,1] \mapsto\langle F(t x+(1-t) y), z\rangle$ is $C$-upper semicontinuous at 0 .

Let us recall the following notions of cone pseudomonotonicity involving a vector-valued function $h$ introduced in [11].

Definition 2.3. A vector-valued function $h: K \times X \rightarrow Z$ is said to be

(i) explicitly $C$-pseudomonotone if, for all $x, y \in K, x \neq y$,

$$
h(x, y) \notin-\operatorname{int}(C) \Rightarrow h(y, x) \in-C ;
$$

(ii) weakly C-pseudomonotone if, for all $x, y \in K, x \neq y$,

$$
h(x, y) \notin-\operatorname{int}(C) \Rightarrow h(y, x) \notin \operatorname{int}(C) .
$$

\section{Remark 2.1.}

(1) When $C=\mathbb{R}^{+}$, both the notions above reduce to the following notion of pseudomonotonicity considered by Komlosi [8]

$$
h(x, y) \geq 0 \Rightarrow h(y, x) \leq 0 .
$$

(2) It is easy to verify that every explicitly $C$-pseudomonotone function is weakly $C$ pseudomonotone. The converse is not true, consider for instance the following example: Take $X=\mathbb{R}, K=\mathbb{R}, Z=\mathbb{R}^{2}, C=\mathbb{R}_{+}^{2}=\left\{(x, y) \in \mathbb{R}^{2}: x \geq 0\right.$ and $\left.y \geq 0\right\}$ and $h: X \times$ $K \rightarrow Z$ is defined by $h(x, y)=(x(y-x), y-x)$. We can verify that $h$ is weakly $C$ pseudomonotone, but it is not explicitly $C$-pseudomonotone, since $h(0,-1) \notin-\operatorname{int}(C)$ and $h(-1,0) \notin-C$.

Definition 2.4. [13, Definition 5.1] A vector-valued function $\varphi: X \rightarrow Z$ is said to be $C$-lower semicontinuous at $x \in X$ iff, for any open neighborhood $V \subset Z$ of $\varphi(x)$, there exists an open neighborhood $U \subset X$ of $x$ such that $\varphi(y) \in V+C$ for all $y \in U$.

$\varphi$ is said to be $C$-lower semicontinuous on $X$ iff it is $C$-lower semicontinuous at each point $x$ of $X$. $\varphi$ said to be $C$-upper semicontinuous on $X$ if $-\varphi$ is $C$-lower semicontinuous on $X$. 
Lemma 2.2. [16] Let $\varphi: X \rightarrow Z$ be a vector-valued function. The following three statements are equivalent:

(i) $\varphi$ is $C$-lower semicontinuous on $X$;

(ii) For all $a \in Z, \varphi^{-1}(a+\operatorname{int}(C))$ is open;

(iii) For each $x_{0} \in X$ and for any $d \in \operatorname{int}(C)$, there exists an open neighborhood $U$ of $x_{0}$ such that $\varphi(x) \in \varphi\left(x_{0}\right)-d+\operatorname{int}(C)$ for all $x \in U$.

\section{EXISTENCE RESULTS FOR BILEVEL VECTOR VARIATIONAL INEQUALITIES}

In this section, we propose a method which consists of reducing the study of the problem of bilevel vector variational inequality (2.2) to that of a one level vector problem. For this, we consider the following problem. Given $\varepsilon>0$, find $x \in K$ such that

$$
\left(\mathscr{P}_{\varepsilon}\right) \quad\langle F(x)+\varepsilon G(x), y-x\rangle+\varphi(y)-\varphi(\bar{x}) \notin-\operatorname{int}(C), \text { for all } y \in K .
$$

Let us denote by $\mathbb{S}_{\varepsilon}$ the solution set of the problem (3.1).

Theorem 3.1. Let $X$ and $Z$ be two Banach spaces, $K$ be a nonempty, closed and convex subset of $X$. Let $F, G: K \rightarrow \mathscr{L}(X, Z)$ be two operators and $\varphi: K \rightarrow Z$ be a vector-valued function. Suppose that the following conditions hold:

(i) $F$ is $C$-monotone and $C$-upper hemicontinuous;

(ii) $\varphi$ is $C$-convex and $C$-lower semicontinuous;

(iii) for each $y \in K, g_{y}(x):=\langle G(x), y-x\rangle$ is $C$-upper semicontinous on $K$;

(iv) (Coercivity condition) There exists a nonempty, compact and convex subset $D_{\varepsilon}$ of $K$ such that either

$\left(\mathscr{C}_{1}\right) \forall y \in K \backslash D_{\mathcal{\varepsilon}}, \exists x \in D_{\varepsilon}$ such that $\langle F(x)+\varepsilon G(y), x-y\rangle+\varphi(x)-\varphi(y) \in-\operatorname{int}(C)$; or

$\left(\mathscr{C}_{2}\right) \exists x_{0} \in D_{\varepsilon}$ such that $\left\langle F(y)+\varepsilon G(y), x_{0}-y\right\rangle+\varphi\left(x_{0}\right)-\varphi(y) \in-\operatorname{int}(C)$, for all $y \in$ $K \backslash D_{\varepsilon}$.

Then, problem $\left(\mathscr{P}_{\varepsilon}\right)$ has a solution $\bar{x}_{\varepsilon}$. Furthermore, $\mathbb{S}_{\mathcal{E}} \subset D_{\varepsilon}$.

Proof. Let $A=\left\{x_{1}, x_{2}, \cdots, x_{n}\right\}$ be a finite subset of $K$, and let $\tilde{D}_{\varepsilon}:=\operatorname{conv}\left(A \cup D_{\varepsilon}\right)$ which is a convex and compact set. For $y \in \tilde{D}_{\varepsilon}$, let us consider the following sets

$$
\begin{aligned}
\mathbb{T}(y) & :=\left\{x \in \tilde{D}_{\varepsilon}:\langle F(x)+\varepsilon G(x), y-x\rangle+\varphi(y)-\varphi(x) \notin-\operatorname{int}(C)\right\}, \\
\mathbb{U}(y) & :=\left\{x \in \tilde{D}_{\varepsilon}:\langle F(y)+\varepsilon G(x), y-x\rangle+\varphi(y)-\varphi(x) \notin-\operatorname{int}(C)\right\} .
\end{aligned}
$$

Note that $\mathbb{T}(y) \subset \mathbb{U}(y)$ since $F$ is $C$-monotone. Furthermore, by using (ii) and (iii), we can verify that $\mathbb{U}(y)$ is closed. Let us verify that $\cap_{y \in \tilde{D}_{\varepsilon}} \mathrm{cl}(\mathbb{T}(y)) \neq \emptyset$. To this aim, let us verify that the family $\{\mathbb{T}(y)\}_{y \in \tilde{D}_{\varepsilon}}$ has the KKM-property. Suppose by contradiction that there exists $\left\{y_{1}, y_{2}, \cdots, y_{p}\right\} \subset$ $\tilde{D}_{\varepsilon}$ such that $\operatorname{conv}\left(\left\{y_{1}, y_{2}, \cdots, y_{p}\right\}\right) \nsubseteq \cup_{i=1}^{p} \mathbb{T}\left(y_{i}\right)$. Hence, there exists $\alpha_{1}, \alpha_{2}, \cdots, \alpha_{p} \geq 0$ with $\sum_{i=1}^{p} \alpha_{i}=1$ such that $\tilde{y}=\sum_{k=1}^{p} \alpha_{k} y_{k} \notin \mathbb{T}\left(y_{i}\right)$, for all $i=1, \cdots, p$. Thus,

$$
\left\langle F(\tilde{y})+\varepsilon G(\tilde{y}), y_{i}-\tilde{y}\right\rangle+\varphi\left(y_{i}\right)-\varphi(\tilde{y}) \in-\operatorname{int}(C), \text { for all } i=1, \cdots, p .
$$

Multiplying the previous relation by $\alpha_{i}$ and taking the sum over $i$, we obtain

$$
\sum_{i=1}^{p} \alpha_{i} \varphi\left(y_{i}\right)-\varphi(\tilde{y}) \in-\operatorname{int}(C) \text {. }
$$


On the other hand, as $\varphi$ is $C$-convex, we derive that

$$
\varphi(\tilde{y})-\sum_{i=1}^{p} \alpha_{i} \varphi\left(y_{i}\right) \in-C .
$$

By adding (3.2) and (3.3), we obtain that

$$
\mathbf{0} \in-C-\operatorname{int}(C)=-\operatorname{int}(C),
$$

which reaches a contradiction. Therefore,

$$
\operatorname{conv}\left(\left\{y_{1}, y_{2}, \cdots, y_{p}\right\}\right) \subset \cup_{i=1}^{p} \mathbb{T}\left(y_{i}\right) \subset \cup_{i=1}^{p} \operatorname{cl}\left(\mathbb{T}\left(y_{i}\right)\right) .
$$

Hence, $\{\operatorname{cl}(\mathbb{T}(y))\}_{y \in \tilde{D}_{\varepsilon}}$ has the KKM-property. Moreover, as $\operatorname{cl}(\mathbb{T}(y))$ is compact for each $y \in$ $\tilde{D}_{\varepsilon}$, we obtain from the Ky Fan lemma [5] that $\cap_{y \in \tilde{D}_{\varepsilon}} \operatorname{cl}(\mathbb{T}(y)) \neq \emptyset$. It follows that $\cap_{y \in \tilde{D}_{\varepsilon}} \mathbb{U}(y) \neq \emptyset$ since $\cap_{y \in \tilde{D}_{\varepsilon}} \operatorname{cl}(\mathbb{T}(y)) \subset \cap_{y \in \tilde{D}_{\varepsilon}} \mathbb{U}(y)$.

Now, let $\tilde{x} \in \cap_{y \in \tilde{D}_{\varepsilon}} \operatorname{cl}(\mathbb{T}(y))$. We verify that $\tilde{x} \in D_{\varepsilon}$. To this aim, we shall use condition (iv). Indeed,

- Suppose that $\left(\mathscr{C}_{1}\right)$ is satisfied and that $\tilde{x} \in K \backslash D_{\varepsilon}$. Then there exists $z \in D_{\varepsilon}$ such that $\langle F(z)+\varepsilon G(\tilde{x}), z-\tilde{x}\rangle+\varphi(z)-\varphi(\tilde{x}) \in-\operatorname{int}(C)$. Hence $\tilde{x} \notin \mathbb{U}(z)$, which is in contradiction with the fact that $\tilde{x} \in \cap_{y \in \tilde{D}_{\varepsilon}} \operatorname{cl}(\mathbb{T}(y)) \subset \cap_{y \in \tilde{D}_{\varepsilon}} \mathbb{U}(y)$.

- Suppose that $\left(\mathscr{C}_{2}\right)$ is satisfied, then $\mathbb{T}\left(x_{0}\right) \subset D_{\varepsilon}$. It follows that $\tilde{x} \in D_{\varepsilon}$.

Consequently, we have proved that for any finite subset $A=\left\{x_{1}, x_{2}, \cdots, x_{n}\right\} \subset K$

$$
\bigcap_{i=1}^{p}\left\{x \in D_{\varepsilon}:\left\langle F\left(x_{i}\right)+\varepsilon G(x), x_{i}-x\right\rangle+\varphi\left(x_{i}\right)-\varphi(x) \notin-\operatorname{int}(C)\right\} \neq \emptyset .
$$

It follows that the family of subsets $\{\mathbb{M}(y)\}_{y \in K} \subset D_{\varepsilon}$, where

$$
\mathbb{M}(y):=\left\{x \in D_{\varepsilon}:\langle F(y)+\varepsilon G(x), y-x\rangle+\varphi(y)-\varphi(x) \notin-\operatorname{int}(C)\right\}
$$

has the finite intersection property. As $D_{\varepsilon}$ is compact, it follows that $\cap_{y \in K} \mathbb{M}(y) \neq \emptyset$. Hence, there exists $\bar{x} \in D_{\varepsilon}$ such that

$$
\langle F(y)+\varepsilon G(\bar{x}), y-\bar{x}\rangle+\varphi(y)-\varphi(\bar{x}) \notin-\operatorname{int}(C), \text { for all } y \in K .
$$

For $y \in K$ and $t \in(0,1]$, let us set

$$
y_{t}=\bar{x}+t(y-\bar{x}) \in K .
$$

As $\varphi$ is $C$-convex, we obtain from (3.4) that

$$
\left\langle F\left(y_{t}\right)+\varepsilon G(\bar{x}), y-\bar{x}\right\rangle+\varphi(y)-\varphi(\bar{x}) \notin-\operatorname{int}(C), \text { for all } y \in K \text {. }
$$

It follows by the $C$-upper hemicontinuity of $F$ that

$$
\langle F(\bar{x})+\varepsilon G(\bar{x}), y-\bar{x}\rangle+\varphi(y)-\varphi(\bar{x}) \notin-\operatorname{int}(C), \text { for all } y \in K \text {. }
$$

Hence, $\bar{x} \in \mathbb{S}_{\varepsilon}$. Now, let us verify that $\mathbb{S}_{\varepsilon} \subset D_{\varepsilon}$. Suppose by contradiction that there exists $\bar{x} \in \mathbb{S}_{\varepsilon}$ such that $\bar{x} \in K \backslash D_{\varepsilon}$. If $\left(\mathscr{C}_{2}\right)$ is satisfied, we deduce that

$$
\left\langle F(\bar{x})+\varepsilon G(\bar{x}), x_{0}-\bar{x}\right\rangle+\varphi\left(x_{0}\right)-\varphi(\bar{x}) \in-\operatorname{int}(C),
$$

which is in contradiction with the fact that $\bar{x} \in \mathbb{S}_{\varepsilon}$. Hence $\bar{x} \in D_{\varepsilon}$. If $\left(\mathscr{C}_{1}\right)$ is satisfied, then there exists $y \in D_{\varepsilon}$ such that

$$
\langle F(y)+\varepsilon G(\bar{x}), y-\bar{x}\rangle+\varphi(y)-\varphi(\bar{x}) \in-\operatorname{int}(C) .
$$


As $F$ is $C$-monotone, we get

$$
\langle F(\bar{x})-F(y), y-\bar{x}\rangle \in-C .
$$

By adding (3.5) and (3.6), we obtain

$$
\langle F(\bar{x})+\varepsilon G(\bar{x}), y-\bar{x}\rangle+\varphi(y)-\varphi(\bar{x}) \in-C-\operatorname{int}(C)=-\operatorname{int}(C) .
$$

This contradicts the fact that $\bar{x} \in \mathbb{S}_{\varepsilon}$. Hence $\bar{x} \in D_{\varepsilon}$, which completes the proof.

Theorem 3.2. Suppose that $X$ is a reflexive Banach space endowed with the weak topology $\sigma\left(X, X^{*}\right)$ and that the conditions (i), (ii) and (iii) of Theorem 3.1 are satisfied. Furthermore, suppose that the following condition holds:

$\left(\mathscr{C}_{0}\right)$ There exists $\boldsymbol{r} \in-\operatorname{int}(C)$ and $x_{0} \in K$ such that for all $s>0$, there exists $R_{s}>0$ satisfying the following: For all $x \in K$ with $\left\|x-x_{0}\right\| \geq R_{s}$, one has $\left\langle G(x), x_{0}-x\right\rangle \in s\left\|x-x_{0}\right\| \boldsymbol{r}-C$.

Then, the problem $\left(\mathscr{P}_{\varepsilon}\right)(3.1)$ has a solution $\bar{x}_{\varepsilon}$.

Proof. We shall apply Theorem 3.1. We need only to verify that the condition $\left(\mathscr{C}_{0}\right)$ implies condition $\left(\mathscr{C}_{2}\right)$, that is, we verify that there exists a nonempty, convex and compact subset $D_{\varepsilon}$ of $K$ with $x_{0} \in D_{\varepsilon}$ such that

$$
\left\langle F(x)+\varepsilon G(x), x_{0}-x\right\rangle+\varphi\left(x_{0}\right)-\varphi(x) \in-\operatorname{int}(C),
$$

for all $x \in K \backslash D_{\varepsilon}$. To this aim, let us consider the following weakly compact set

$$
B=\left\{x \in K:\left\|x-x_{0}\right\| \leq \alpha\right\},
$$

where $\alpha>0$, and the vector-valued function $h_{x_{0}}: X \rightarrow Z$ defined by

$$
h_{x_{0}}(x)=\left\langle F\left(x_{0}\right), x-x_{0}\right\rangle+\varphi(x)-\varphi\left(x_{0}\right) .
$$

As $h_{x_{0}}(\cdot)$ is $C$-lower semicontinuous, then, according to [10, Lemma 7], there exists $\mathbf{r}_{0} \in Z$ such that

$$
h_{x_{0}}(x)-\mathbf{r}_{0} \in \operatorname{int}(C), \quad \text { for all } x \in B .
$$

On the other hand, as $-\mathbf{r} \in \operatorname{int}(C)$, we have $-\alpha \mathbf{r} \in \operatorname{int}(C)$. Hence $\mathbf{0} \in \alpha \mathbf{r}+\operatorname{int}(C)$. It follows that there exists $q \in(0,1)$ such that, for all $t \in(0, q), t \mathbf{r}_{0} \in \alpha \mathbf{r}+\operatorname{int}(C)$. Hence, for $s>0$ such that $s>\frac{1}{q}$, we have

$$
\mathbf{r}_{0} \in \alpha s \mathbf{r}+\operatorname{int}(C)
$$

Now, let $x \in K \backslash B$ and set

$$
\tilde{x}:=\frac{\alpha}{\left\|x-x_{0}\right\|} x+\left(1-\frac{\alpha}{\left\|x-x_{0}\right\|}\right) x_{0} \in B .
$$

From (3.7), we get $h_{x_{0}}(\tilde{x})-\mathbf{r}_{0} \in \operatorname{int}(C)$. As $\varphi$ is $C$-convex, it follows

$$
\left\langle F\left(x_{0}\right), x-x_{0}\right\rangle+\varphi(x)-\varphi\left(x_{0}\right)-\frac{\left\|x-x_{0}\right\|}{\alpha} \mathbf{r}_{0} \in \operatorname{int}(C) .
$$

Since $F$ is $C$-monotone, we derive from the previous relation that

$$
\left\langle F(x), x_{0}-x\right\rangle+\varphi\left(x_{0}\right)-\varphi(x)+\frac{\left\|x-x_{0}\right\|}{\alpha} \mathbf{r}_{0} \in-\operatorname{int}(C) .
$$

Consequently, for any $x \in K \backslash B$ and $\varepsilon \in(0,1)$, we have

$$
\left\langle F(x)+\varepsilon G(x), x_{0}-x\right\rangle+\varphi\left(x_{0}\right)-\varphi(x)+\frac{\left\|x-x_{0}\right\|}{\alpha} \mathbf{r}_{0} \in \varepsilon\left\langle G(x), x_{0}-x\right\rangle-\operatorname{int}(C) .
$$


On the other hand, for $\varepsilon \in(0,1)$ and $s>0$ such that $s>\frac{1}{q}$, let us set $\tilde{s}=\frac{s}{\varepsilon}>0$. By using $\left(\mathscr{C}_{0}\right)$, there exists $\beta_{\tilde{s}}>0$ such that for all $x \in K$ satisfying $\left\|x-x_{0}\right\| \geq \beta_{\tilde{s}}$, one has

$$
\left\langle G(x), x_{0}-x\right\rangle \in \tilde{s}\left\|x-x_{0}\right\| \mathbf{r}-C .
$$

It follows that for $s>\frac{1}{q}, \varepsilon \in(0,1)$ and $x \in K$ satisfying $\left\|x-x_{0}\right\|>\beta_{\tilde{s}}$, one has

$$
\varepsilon\left\langle G(x), x_{0}-x\right\rangle \in s\left\|x-x_{0}\right\| \mathbf{r}-C .
$$

Therefore, from (3.9) and (3.10), we deduce that for $s>\frac{1}{q}, \varepsilon \in(0,1)$ and $x \in K \backslash B$ such that $\left\|x-x_{0}\right\|>\beta_{\tilde{s}}$ the following relation is satisfied

$$
\left\langle F(x)+\varepsilon G(x), x_{0}-x\right\rangle+\varphi\left(x_{0}\right)-\varphi(x) \in \frac{\left\|x-x_{0}\right\|}{\alpha}\left(\alpha s \mathbf{r}-\mathbf{r}_{0}\right)-\operatorname{int}(C) .
$$

Let $R_{\varepsilon}=\max \left\{\alpha, \beta_{\tilde{s}}\right\}$ and let us set $D_{\varepsilon}:=\left\{x \in K:\left\|x-x_{0}\right\| \leq R_{\varepsilon}\right\}$, which is a nonempty, convex and compact subset of $K$. Then, from (3.8) and (3.11), we deduce that

$$
\left\langle F(x)+\varepsilon G(x), x_{0}-x\right\rangle+\varphi\left(x_{0}\right)-\varphi(x) \in-\operatorname{int}(C), \quad \text { for all } x \in K \backslash D_{\varepsilon} .
$$

This completes the proof.

Remark 3.1. If $Z=\mathbb{R}$ and $C=\left[0,+\infty\left[\right.\right.$, the condition $\left(\mathscr{C}_{0}\right)$ is equivalent to the following condition:

$$
\frac{\left\langle G(x), x-x_{0}\right\rangle}{\left\|x-x_{0}\right\|} \longrightarrow+\infty \text {, when }\left\|x-x_{0}\right\| \longrightarrow+\infty \text {. }
$$

Theorem 3.3. Let $X$ and $Z$ be two Banach spaces, $K$ be a nonempty, closed and convex subset of $X$. Let $F, G: K \rightarrow \mathscr{L}(X, Z)$ be two operators and $\varphi: K \rightarrow Z$ be a vector-valued function. Suppose that the following conditions hold:

(i) $F$ is $C$-monotone and $C$-upper hemicontinuous;

(ii) $\varphi$ is $C$-convex and $C$-lower semicontinuous;

(iii) For each $x, y \in K, h(x, y):=\langle F(x), y-x\rangle+\varphi(y)-\varphi(x)$ is explicitly $C$-pseudomonotone;

(iv) for each $y \in K$ fixed, $g_{y}(x):=\langle G(x), y-x\rangle$ is $C$-upper semicontinous on $K$;

(v) for each $\varepsilon>0$, the problem $\left(\mathscr{P}_{\varepsilon}\right)(3.1)$ has a solution $\bar{x}_{\varepsilon}$.

Then, every cluster point $\bar{x}$ of $\left\{\bar{x}_{\varepsilon}\right\}_{\varepsilon>0}$ is a solution of the bilevel problem (2.2).

Proof. Let $\left\{\bar{x}_{\varepsilon}\right\}_{\varepsilon>0} \subset K$ such that $\bar{x}_{\varepsilon} \in \mathbb{S}_{\varepsilon}$ and let $\bar{x}$ be a cluster point of $\left\{\bar{x}_{\varepsilon}\right\}_{\varepsilon>0}$.

Step 1. Let us verify that $\bar{x} \in \mathbb{S}$. To this aim, as $\bar{x}_{\varepsilon} \in \mathbb{S}_{\varepsilon}$, it follows that

$$
\left\langle F\left(\bar{x}_{\varepsilon}\right)+\varepsilon G\left(\bar{x}_{\varepsilon}\right), y-\bar{x}_{\varepsilon}\right\rangle+\varphi(y)-\varphi\left(\bar{x}_{\varepsilon}\right) \notin-\operatorname{int}(C), \text { for all } y \in K .
$$

On the other hand, since $F$ is $C$-monotone, we have

$$
\left\langle F(y)-F\left(\bar{x}_{\varepsilon}\right), y-\bar{x}_{\varepsilon}\right\rangle \in C .
$$

From (3.12) and (3.13), we deduce by using Lemma 2.1, that

$$
\left\langle F(y)+\varepsilon G\left(\bar{x}_{\varepsilon}\right), y-\bar{x}_{\varepsilon}\right\rangle+\varphi(y)-\varphi\left(\bar{x}_{\varepsilon}\right) \notin-\operatorname{int}(C) \text {, for all } y \in K .
$$

We claim that $\langle F(y), y-\bar{x}\rangle+\varphi(y)-\varphi(\bar{x}) \notin-\operatorname{int}(C)$, for all $y \in K$. Indeed, suppose by contradiction that there exists $\tilde{y} \in K$ such that

$$
\langle F(\tilde{y}), \tilde{y}-\bar{x}\rangle+\varphi(\tilde{y})-\varphi(\bar{x}) \in-\operatorname{int}(C) .
$$


Then there exists $\mathbf{r} \in \operatorname{int}(C)$ such that

$$
\langle F(\tilde{y}), \tilde{y}-\bar{x}\rangle+\varphi(\tilde{y})-\varphi(\bar{x})+\mathbf{r} \in-\operatorname{int}(C) .
$$

As $\frac{1}{2} \mathbf{r}-\operatorname{int}(C)$ is open and $\mathbf{0} \in \frac{1}{2} \mathbf{r}-\operatorname{int}(C)$, there exists $k_{1}>0$ such that

$$
\varepsilon\langle G(\bar{x}), \tilde{y}-\bar{x}\rangle \in \frac{1}{2} \mathbf{r}-\operatorname{int}(C), \text { for all } \varepsilon \in\left[0, k_{1}\right) .
$$

On the other hand, as $g_{\tilde{y}}(x):=\langle G(x), \tilde{y}-x\rangle$ is $C$-upper semicontinous on $K$ and $\varphi$ is $C$-lower semicontinuous on $K$, it follows that the vector-valued function $h_{\tilde{y}}: K \rightarrow Z$ defined by $h_{\tilde{y}}(x)=\langle F(\tilde{y})+\varepsilon G(x), \tilde{y}-x\rangle+\varphi(\tilde{y})-\varphi(x)$ is $C$-upper semicontinuous on $K$. Therefore, there exists $k_{2}>0$ and a neighborhood $U$ of $\bar{x}$ such that $\bar{x}_{\varepsilon} \in U$ for $\varepsilon \in\left[0, k_{2}\right)$ and $h_{\tilde{y}}(x) \in h_{\tilde{y}}(\bar{x})+\frac{1}{2} \mathbf{r}-\operatorname{int}(C)$ for all $x \in U$. Hence,

$$
h_{\tilde{y}}\left(\bar{x}_{\varepsilon}\right) \in h_{\tilde{y}}(\bar{x})+\frac{1}{2} \mathbf{r}-\operatorname{int}(C), \text { for all } \varepsilon \in\left[0, k_{2}\right) .
$$

Let $k=\min \left\{k_{1}, k_{2}\right\}$. From (3.15), (3.16) and (3.17), we deduce that

$$
\left\langle F(\tilde{y})+\varepsilon G\left(\bar{x}_{\varepsilon}\right), \tilde{y}-\bar{x}_{\varepsilon}\right\rangle+\varphi(\tilde{y})-\varphi\left(\bar{x}_{\varepsilon}\right) \in-\operatorname{int}(C), \text { for } \varepsilon \in[0, k),
$$

which is in contradiction with (3.14). Thus,

$$
\langle F(y), y-\bar{x}\rangle+\varphi(y)-\varphi(\bar{x}) \notin-\operatorname{int}(C), \text { for all } y \in K .
$$

For $y \in K$ and $t \in(0,1]$, let $y_{t}=\bar{x}+t(y-\bar{x}) \in K$. From (3.18) and the $C$-convexity of $\varphi$, we derive that

$$
\left\langle F\left(y_{t}\right), y-\bar{x}\right\rangle+\varphi(y)-\varphi(\bar{x}) \notin-\operatorname{int}(C) .
$$

By using the $C$-upper hemicontinuity of $F$, we deduce that

$$
\langle F(\bar{x}), y-\bar{x}\rangle+\varphi(y)-\varphi(\bar{x}) \notin-\operatorname{int}(C),
$$

for all $y \in K$. Hence, $\bar{x} \in \mathbb{S}$.

Step 2. We show that $\bar{x}$ is a solution of the bilevel problem (2.2). Let $w \in \mathbb{S}$ and $\bar{x}_{\varepsilon} \in \mathbb{S}_{\varepsilon}$. Then

$$
\left\langle F\left(\bar{x}_{\varepsilon}\right)+\varepsilon G\left(\bar{x}_{\varepsilon}\right), w-\bar{x}_{\varepsilon}\right\rangle+\varphi(w)-\varphi\left(\bar{x}_{\varepsilon}\right) \notin-\operatorname{int}(C) .
$$

Let us verify that $\left\langle G\left(\bar{x}_{\varepsilon}\right), w-\bar{x}_{\varepsilon}\right\rangle \notin-\operatorname{int}(C)$. Suppose by contradiction that

$$
\left\langle G\left(\bar{x}_{\varepsilon}\right), w-\bar{x}_{\varepsilon}\right\rangle \in-\operatorname{int}(C) .
$$

As $\left\langle F(w), \bar{x}_{\varepsilon}-w\right\rangle+\varphi\left(\bar{x}_{\varepsilon}\right)-\varphi(w) \notin-\operatorname{int}(C)$, it follows from condition (iii) that

$$
\left\langle F\left(\bar{x}_{\varepsilon}\right), \bar{x}_{\varepsilon}-w\right\rangle+\varphi\left(\bar{x}_{\varepsilon}\right)-\varphi(w) \in C .
$$

Hence, from (3.19) and (3.21), it follows from Lemma 2.1, that

$$
\varepsilon\left\langle G\left(\bar{x}_{\varepsilon}\right), w-\bar{x}_{\varepsilon}\right\rangle \notin-\operatorname{int}(C),
$$

which contradicts (3.20). Thus, $\left\langle G\left(\bar{x}_{\varepsilon}\right), w-\bar{x}_{\varepsilon}\right\rangle \notin-\operatorname{int}(C)$. As $\bar{x}$ is a cluster point of $\left\{\bar{x}_{\varepsilon}\right\}_{\varepsilon>0}$ and the vector-valued function $g_{w}(x)=\langle G(x), w-x$ is $C$-upper semicontinuous at $\bar{x}$, we derive that $\langle G(\bar{x}), w-\bar{x}\rangle \notin-\operatorname{int}(C)$. Therefore, $\bar{x}$ is a solution of the bilevel problem (2.2). 
Example 3.1. Let $X=\mathbb{R}, Z=\mathbb{R}^{2}, C=\mathbb{R}_{+}^{2}=\left\{(x, y) \in \mathbb{R}^{2}: x \geq 0\right.$ and $\left.y \geq 0\right\}$ and $K=[0,+\infty)$. Consider the operator $F: K \rightarrow \mathscr{L}(X, Z)$ defined by $\langle F(x), y-x\rangle=(x(y-x), y-x)$ and the function vector-valued function $\varphi: K \rightarrow Z$ defined by $\varphi(x)=(x, x)$. Then one can easily verify that $F$ and $\varphi$ satisfy the conditions (i)-(iii) of Theorem 3.3.

Theorem 3.4. Suppose that the conditions (i), (ii), (iii) and (iv) of Theorem 3.3 are satisfied, and the following condition holds:

$(\mathscr{C})$ There exist a nonempty, convex and compact set $D \subset X$ and $x_{0} \in D \cap K$ such that for all $x \in K \backslash D$, we have $\left\langle F(x)+\varepsilon G(x), x_{0}-x\right\rangle+\varphi\left(x_{0}\right)-\varphi(x) \in-\operatorname{int}(C)$.

Then, bilevel problem (2.2) has a solution in $D$.

Proof. By using Theorem 3.1, we deduce that, for each $\varepsilon>0$, problem $(\mathscr{P})_{\varepsilon}(3.1)$ has a solution $\bar{x}_{\varepsilon}$ and $\mathbb{S}_{\varepsilon} \subset D$. It follows that $\left\{x_{\varepsilon}\right\}_{\varepsilon>0}$ has a cluster point. Therefore, by using Theorem 3.3, we conclude that bilevel problem (2.2) has a solution in $D$.

Theorem 3.5. Let $X$ be a reflexive Banach space endowed with the weak topology $\sigma\left(X, X^{*}\right)$. Suppose that the conditions (i), (ii), (iii) and (iv) of Theorem 3.3 are satisfied, and the following conditions hold: there exists $\boldsymbol{r} \in \operatorname{int}(C)$ and $x_{0} \in K$ such that

$\left(\mathscr{D}_{1}\right)$ For a certain $R_{0}>0$ and any $x \in K$ with $\left\|x-x_{0}\right\|>R_{0}$, one has

$$
\left\langle G(x), x_{0}-x\right\rangle \in\left\|x-x_{0}\right\| \boldsymbol{r}-C
$$

$\left(\mathscr{D}_{2}\right)$ For all $s>0$, there exists $R_{s}>0$ such that for $x \in K$ with $\left\|x-x_{0}\right\|>R_{s}$ one has

$$
\left\langle F(x), x-x_{0}\right\rangle+\varphi(x)-\varphi\left(x_{0}\right) \in s\left\|x-x_{0}\right\| \boldsymbol{r}+C .
$$

Then, problem (2.2) has a solution.

Proof. Let us verify that there exists a nonempty, compact and convex subset $D$ of $K$, which does depend on $\varepsilon>0$ (sufficiently small) such that

$$
\left\langle F(x)+\varepsilon G(x), x_{0}-x\right\rangle+\varphi\left(x_{0}\right)-\varphi(x) \in-\operatorname{int}(C), \quad \text { for all } x \in K \backslash D .
$$

From $\left(\mathscr{D}_{1}\right)$, there exists $R_{0}>0$ such that $\left\langle G(x), x_{0}-x\right\rangle \in\left\|x-x_{0}\right\| \mathbf{r}-C$, for $x \in K$ satisfying $\left\|x-x_{0}\right\|>R_{0}$. Hence, for $\varepsilon \in(0,1)$ and $x \in K$ satisfying $\left\|x-x_{0}\right\|>R$, one has

$$
\varepsilon\left\langle G(x), x_{0}-x\right\rangle \in \varepsilon\left\|x-x_{0}\right\| \mathbf{r}-C .
$$

By considering $s=1$ in $\left(\mathscr{D}_{2}\right)$, there exists $R_{1}>0$ such that

$$
\left\langle F(x), x-x_{0}\right\rangle+\varphi(x)-\varphi\left(x_{0}\right) \in\left\|x-x_{0}\right\| \mathbf{r}+C, \quad \text { for all } x \in K \text { satisfying }\left\|x-x_{0}\right\|>R_{1} .
$$

Let us set $R=\max \left\{R_{0}, R_{1}\right\}$. By using (3.23) and (3.24), we get, for all $x \in K$ such that $\| x-$ $x_{0} \|>R$,

$$
\left\langle F(x)+\varepsilon G(x), x_{0}-x\right\rangle+\varphi\left(x_{0}\right)-\varphi(x) \in-(1-\varepsilon)\left\|x-x_{0}\right\| \mathbf{r}-C .
$$

Since $\varepsilon \in(0,1)$ and $\mathbf{r} \in \operatorname{int}(C)$, we deduce that

$$
-(1-\varepsilon)\left\|x-x_{0}\right\| \mathbf{r} \in-\operatorname{int}(C), \quad \text { for all } \varepsilon \in(0,1) .
$$

By taking $D=\left\{x \in K:\left\|x-x_{0}\right\| \leq R\right\}$ (which does not depend on $\varepsilon$ ), we derive from (3.25) that

$$
\left\langle F(x)+\varepsilon G(x), x_{0}-x\right\rangle+\varphi\left(x_{0}\right)-\varphi(x) \in-\operatorname{int}(C), \quad \text { for all } x \in K \backslash D .
$$

We conclude the desired conclusion by using Theorem 3.4. 


\section{THE VECTOR AUXILIARY PRINCIPLE FOR BILEVEL VECTOR VARIATIONAL INEQUALITIES}

Let $T: X \rightarrow \mathscr{L}(X, Z)$ be a linear continuous operator, $w \in K, \varepsilon>0$ and $\rho>0$. In order to approximate the solutions of bilevel problem (2.2), we consider the following auxiliary vector variational inequality problem (for short, $(\mathrm{AVVIP})_{\rho, \varepsilon}$ ): Find $\bar{x} \in K$ such that

$$
\rho[\langle F(\bar{x})+\varepsilon G(\bar{x}), y-\bar{x}\rangle+\varphi(y)-\varphi(\bar{x})]+\langle T(y)-T(\bar{x}), \bar{x}-w\rangle \notin-\operatorname{int}(C), \quad \text { for all } y \in K .
$$

Definition 4.1. Let $T: X \rightarrow \mathscr{L}(X, Z)$ be an operator and $\mathbf{a} \in \operatorname{int}(C)$.

(a) $T$ is said to be $\boldsymbol{a}$-C-strongly positive with modulus a if

$$
\langle T(x), x\rangle-\|x\|^{2} \mathbf{a} \in C, \quad \text { for all } x \in X .
$$

(b) $T$ is said to be $\boldsymbol{a}$-C-strongly monotone with modulus a if

$$
\langle T(x)-T(y), x-y\rangle-\|x-y\|^{2} \mathbf{a} \in C, \quad \text { for all } x, y \in X .
$$

Remark 4.1. It is easy to see that if a linear operator $T: X \rightarrow \mathscr{L}(X, Z)$ is a- $C$-strongly positive then it is a- $C$-strongly monotone.

Theorem 4.1. Suppose that $X$ is a reflexive Banach space and $K$ is a nonempty, closed and convex subset of $X$. Let $F, G: K \rightarrow \mathscr{L}(X, Z)$ be two operators and $T: X \rightarrow \mathscr{L}(X, Z)$ be a bounded linear operator. Assume that there exists $\varpi \in \operatorname{int}\left(C^{*}\right)$ such that $\langle\varpi, z\rangle \geq 0$ for all $z \notin-\operatorname{int}(C)$. Suppose that the following conditions hold:

(i) $F$ and $G$ are $C$-monotone and $C$-upper hemicontinuous;

(ii) $\varphi$ is $C$-convex and $C$-lower semicontinuous;

(iii) $T$ is $\boldsymbol{a}$-C-strongly positive with modulus $\boldsymbol{a} \in \operatorname{int}(C)$.

Then, for each $w \in X$, problem $(A V E P)_{\rho, \varepsilon}(4.1)$ has a unique solution $\bar{x} \in K$.

Proof. Let us consider the operator $F_{\rho, \varepsilon}: K \rightarrow \mathscr{L}(X, Z)$ defined by $F_{\rho, \varepsilon}(x)=\rho(F(x)+\varepsilon G(x))$ and the vector-valued function $\varphi_{\rho}: K \rightarrow Z$ defined by $\varphi_{\rho}(x)=\rho \varphi(x)$. It is easy to verify that the operator $F_{\rho, \varepsilon}$ is $C$-monotone and $C$-upper hemicontinuous and that $\varphi_{\rho}$ is $C$-convex and $C$ lower semicontinuous. By using these notations, we can rewrite the problem (AVEP) $)_{\rho, \varepsilon}(4.1)$ as the following: Find $\bar{x} \in K$ such that

$$
\left\langle F_{\rho, \varepsilon}(\bar{x}), y-\bar{x}\right\rangle+\varphi_{\rho}(y)-\varphi_{\rho}(\bar{x})+\langle T(y)-T(\bar{x}), \bar{x}-w\rangle \notin-\operatorname{int}(C), \quad \text { for all } y \in K .
$$

We shall proceed by the three steps below.

Step 1. First, let us verify that there exists a compact convex subset $D_{\rho, \varepsilon}$ of $K$ and $x_{0} \in K$, such that

$$
\left\langle F_{\rho, \varepsilon}(x), x_{0}-x\right\rangle+\varphi_{\rho}\left(x_{0}\right)-\varphi_{\rho}(x)+\left\langle T\left(x_{0}\right)-T(x), x-w\right\rangle \in-\operatorname{int}(C), \quad \text { for all } x \in K \backslash D_{\rho, \varepsilon} .
$$

To this aim, as $F_{\rho, \varepsilon}$ is $C$-monotone, it suffices to verify, by following a similar procedure to the one used in the proof of Theorem 3.2, that there exist $\mathbf{r} \in-\operatorname{int}(C)$ and $x_{0} \in K$ such that for all $s>0$, there exists $R_{S}>0$ satisfying the following condition:

$$
\left\langle T\left(x_{0}\right)-T(x), x-w\right\rangle \in s\left\|u-v_{0}\right\| \mathbf{r}-C \text {, for all } x \in K \text { with }\left\|x-x_{0}\right\|>R_{s} .
$$


Let $x_{0} \in K$ be arbitrary and set $\mathbf{r}:=-\mathbf{a} \in-\operatorname{int}(C)$. Note that

$$
\left\langle T\left(x_{0}\right)-T(x), x-w\right\rangle=-\left\langle T\left(x-x_{0}\right), x-x_{0}\right\rangle+\left\langle T\left(x_{0}\right)-T(x), x_{0}-w\right\rangle
$$

Therefore, for $s>0$, we have

$$
\begin{aligned}
& s\left\|x-x_{0}\right\| \mathbf{r}-\left\langle T\left(x_{0}\right)-T(x), x-w\right\rangle \\
& =s\left\|x-x_{0}\right\| \mathbf{r}+\left\langle T\left(x-x_{0}\right), x-x_{0}\right\rangle+\left\langle T\left(x-x_{0}\right), x_{0}-w\right\rangle \\
& =\left[\left\langle T\left(x-x_{0}\right), x-x_{0}\right\rangle-\mathbf{a}\left\|x-x_{0}\right\|^{2}\right] \\
& \quad+\mathbf{a}\left\|x-x_{0}\right\|^{2}-s\left\|x-x_{0}\right\| \mathbf{a}+\left\langle T\left(x-x_{0}\right), x_{0}-w\right\rangle .
\end{aligned}
$$

Let us set $\psi(x):=\left\langle T\left(x-x_{0}\right), x_{0}-w\right\rangle$. As $T$ is a-C-strongly positive, it follows from (4.5) that

$$
s\left\|x-x_{0}\right\| \mathbf{r}-\left\langle T\left(x_{0}\right)-T(x), x-w\right\rangle \in\left\|x-x_{0}\right\|^{2} \mathbf{a}-s\left\|x-x_{0}\right\| \mathbf{a}+\psi(x)+C .
$$

As $T: X \rightarrow \mathscr{L}(X, Z)$ is linear continuous, it follows that $\psi(\cdot)$ is continuous from $X$ to $Z$, and therefore it is $C$-lower semicontinuous. Hence, for $\alpha>0$ there exists $\mathbf{r}_{0} \in Z$ such that

$$
\psi(y)-\mathbf{r}_{0} \in \operatorname{int}(C), \quad \text { for all } y \in B:=\left\{x \in K:\left\|x-x_{0}\right\| \leq \alpha\right\} .
$$

On the other hand, we have $\mathbf{a} \in \operatorname{int}(C)$. Hence $\alpha \mathbf{a} \in \operatorname{int}(C)$ and $\mathbf{0} \in-\alpha \mathbf{a}+\operatorname{int}(C)$. Thus, there exists $q \in(0,1)$ such that, for all $t \in(0, q), t \mathbf{r}_{0} \in-\alpha \mathbf{a}+\operatorname{int}(C)$. Therefore,

$$
\mathbf{r}_{0} \in-s \alpha \mathbf{a}+\operatorname{int}(C), \quad \text { for } s>\frac{1}{q} \text {. }
$$

Now, let $x \in K \backslash B$ and $y:=\frac{\alpha}{\left\|x-x_{0}\right\|} x+\left(1-\frac{\alpha}{\left\|x-x_{0}\right\|}\right) x_{0} \in B$. Note that

$$
\psi(y)=\frac{\alpha}{\left\|x-x_{0}\right\|} \psi(x)+\left(1-\frac{\alpha}{\left\|x-x_{0}\right\|}\right) \psi\left(x_{0}\right)=\frac{\alpha}{\left\|x-x_{0}\right\|} \psi(x) .
$$

From (4.7), we deduce that $\frac{\alpha}{\left\|x-x_{0}\right\|} \psi(x)-\mathbf{r}_{0} \in \operatorname{int}(C)$. Therefore,

$$
\psi(x) \in \frac{\left\|x-x_{0}\right\|}{\alpha} \mathbf{r}_{0}+\operatorname{int}(C), \quad \text { for all } x \in K \backslash B .
$$

Thus, from (4.6) and (4.9), we deduce

$$
\begin{aligned}
& s\left\|x-x_{0}\right\| \mathbf{r}-\left\langle T\left(x_{0}\right)-T(x), x-w\right\rangle \\
& \in\left[\left\|x-x_{0}\right\|-2 s\right]\left\|x-x_{0}\right\| \mathbf{a}+\frac{\left\|x-x_{0}\right\|}{\alpha}\left[\mathbf{r}_{0}+s \alpha \mathbf{a}\right]+\operatorname{int}(C) .
\end{aligned}
$$

By considering $R_{s}:=\max \{2 s, \alpha\}$, we deduce from (4.8) and (4.10) that for $x \in K$ such that $\left\|x-x_{0}\right\| \geq R_{s}$, we have

$$
s\left\|x-x_{0}\right\| \mathbf{r}-\left\langle T\left(x_{0}\right)-T(x), x-w\right\rangle \in \operatorname{int}(C) .
$$

Hence, we obtain relation (4.4).

Step 2. In this second step, we show that the problem (AVEP) $\rho_{\rho, \varepsilon}(4.1)$ has a solution. To this aim, let $A=\left\{x_{1}, x_{2}, \cdots, x_{n}\right\}$ be a finite subset of $K$, and let $\tilde{D}_{\rho, \varepsilon}:=\operatorname{conv}\left(A \cup D_{\rho, \varepsilon}\right)$ which is a convex and compact set. We follow the same procedure used in the proof of Theorem 3.1 by considering, for $y \in \tilde{D}_{\varepsilon}$, the sets below

$$
\tilde{\mathbb{T}}(y):=\left\{x \in \tilde{D}_{\rho, \varepsilon}:\left\langle F_{\rho, \varepsilon}(x), y-x\right\rangle+\varphi_{\rho}(y)-\varphi_{\rho}(x)+\langle T(y)-T(x), x-w\rangle \notin-\operatorname{int}(C)\right\},
$$


$\tilde{\mathbb{U}}(y):=\left\{x \in \tilde{D}_{\rho, \varepsilon}:\left\langle F_{\rho, \varepsilon}(y), y-x\right\rangle+\varphi_{\rho}(y)-\varphi_{\rho}(x)+\langle T(y)-T(x), x-w\rangle \notin-\operatorname{int}(C)\right\}$.

The rest of the proof is similar to the one of Theorem 3.1, where $\mathbb{T}(y)$ is replaced by $\tilde{\mathbb{T}}(y)$, and $\mathbb{U}(y)$ is replaced by $\tilde{U}(y)$.

Step 3. We verify that the solution of $(\mathrm{AVEP})_{\rho, \varepsilon}$ is unique. Suppose that the problem (4.1) has two solutions $x_{1}$ and $x_{2}$. Then,

$$
\begin{aligned}
& \left\langle F_{\rho, \varepsilon}\left(x_{1}\right), y-x_{1}\right\rangle+\varphi_{\rho}(y)-\varphi_{\rho}\left(x_{1}\right)+\left\langle T(y)-T\left(x_{1}\right), x_{1}-w\right\rangle \notin-\operatorname{int}(C), \quad \text { for all } y \in K, \\
& \quad \text { and } \\
& \left\langle F_{\rho, \varepsilon}\left(x_{2}\right), y-x_{2}\right\rangle+\varphi_{\rho}(y)-\varphi_{\rho}\left(x_{2}\right)+\left\langle T(y)-T\left(x_{2}\right), x_{2}-w\right\rangle \notin-\operatorname{int}(C), \quad \text { for all } y \in K .
\end{aligned}
$$

Taking $y=x_{2}$ in (4.11) and $y=x_{1}$ in (4.12), we obtain

$$
\left\langle F_{\rho, \varepsilon}\left(x_{1}\right), x_{2}-x_{1}\right\rangle+\varphi_{\rho}\left(x_{2}\right)-\varphi_{\rho}\left(x_{1}\right)+\left\langle T\left(x_{2}\right)-T\left(x_{1}\right), x_{1}-w\right\rangle \notin-\operatorname{int}(C),
$$

and

$$
\left\langle F_{\rho, \varepsilon}\left(x_{2}\right), x_{1}-x_{2}\right\rangle+\varphi_{\rho}\left(x_{1}\right)-\varphi_{\rho}\left(x_{2}\right)+\left\langle T\left(x_{1}\right)-T\left(x_{2}\right), x_{2}-w\right\rangle \notin-\operatorname{int}(C) .
$$

It follows that

$$
\left\langle\varpi,\left\langle F_{\rho, \varepsilon}\left(x_{1}\right), x_{2}-x_{1}\right\rangle+\varphi_{\rho}\left(x_{2}\right)-\varphi_{\rho}\left(x_{1}\right)+\left\langle T\left(x_{2}\right)-T\left(x_{1}\right), x_{1}-w\right\rangle\right\rangle \geq 0,
$$

and

$$
\left\langle\varpi,\left\langle F_{\rho, \varepsilon}\left(x_{2}\right), x_{1}-x_{2}\right\rangle+\varphi_{\rho}\left(x_{1}\right)-\varphi_{\rho}\left(x_{2}\right)+\left\langle T\left(x_{1}\right)-T\left(x_{2}\right), x_{2}-w\right\rangle\right\rangle \geq 0 .
$$

Adding inequalities (4.13) and (4.14), we get

$$
\left\langle\varpi,\left\langle F_{\rho, \varepsilon}\left(x_{1}\right)-F_{\rho, \varepsilon}\left(x_{2}\right), x_{2}-x_{1}\right\rangle\right\rangle+\left\langle\varpi,\left\langle T\left(x_{2}\right)-T\left(x_{1}\right), x_{1}-x_{2}\right\rangle\right\rangle \geq 0 .
$$

Since $F_{\rho, \varepsilon}$ is $C$-monotone, it follows that $\left\langle F_{\rho, \varepsilon}\left(x_{1}\right)-F_{\rho, \varepsilon}\left(x_{2}\right), x_{1}-x_{2}\right\rangle \in C$. Hence

$$
\left\langle\varpi,\left\langle F_{\rho, \varepsilon}\left(x_{1}\right)-F_{\rho, \varepsilon}\left(x_{2}\right), x_{2}-x_{1}\right\rangle\right\rangle \leq 0
$$

Thus, from (4.15), we deduce that

$$
\left\langle\varpi,\left\langle T\left(x_{2}\right)-T\left(x_{1}\right), x_{1}-x_{2}\right\rangle\right\rangle \geq 0 .
$$

On the other hand, from Remark 4.1, we have that $T$ is a- $C$-strongly monotone, and hence

$$
\left\langle T\left(x_{2}\right)-T\left(x_{1}\right), x_{2}-x_{1}\right\rangle-\left\|x_{2}-x_{1}\right\|^{2} \mathbf{a} \in C .
$$

Therefore,

$$
\left\langle\varpi,\left\langle T\left(x_{2}\right)-T\left(x_{1}\right), x_{1}-x_{2}\right\rangle\right\rangle \leq-\left\|x_{2}-x_{1}\right\|^{2}\langle\varpi, \mathbf{a}\rangle .
$$

In view of (4.16), we deduce that $-\left\|x_{2}-x_{1}\right\|^{2}\langle\varpi, \mathbf{a}\rangle \geq 0$. Since $\langle\varpi, \mathbf{a}\rangle>0$, it follows that $x_{1}=x_{2}$.

Remark 4.2. In the previous results, we have used the following condition:

There exists $\varpi \in \operatorname{int}\left(C^{*}\right)$ such that $\langle\varpi, z\rangle \geq 0$ for all $z \notin-\operatorname{int}(C)$.

We give the following example where this condition is satisfied: Let $Z=\mathbb{R}^{2}$ and $C=\mathbb{R}_{+} \times \mathbb{R}=$ $\{(x, y): x \geq 0\}$. Then, $\operatorname{int}(C)=\{(x, y): x>0\}$. Let $s=\left(s_{1}, 0\right)$ with $s_{1}>0$. Then $s \in \operatorname{int}\left(C^{*}\right)$ and $\langle s, z\rangle \geq 0$ for all $z \notin-\operatorname{int}(C)$. When $Z=\mathbb{R}$ and $C=[0,+\infty)$, this condition is trivial. We point 
out this condition has been used in several works, see, for instance, [17, Theorem 3.6,Theorem 4.2].

Now, we present an iterative method to approximate the solution of bilevel problem (2.2) and study its convergence.

Algorithm 4.1. Take $\left.\left\{\varepsilon_{n}\right\}_{n \in \mathbb{N}} \subset\right] 0,+\infty\left[\right.$ and $\left.\left\{\rho_{n}\right\}_{n \in \mathbb{N}} \subset\right] 0,+\infty\left[\right.$ such that $\left\{\varepsilon_{n}\right\}_{n \in \mathbb{N}}$ is decreasing to 0 and $\left\{\rho_{n}\right\}_{n \in \mathbb{N}}$ is increasing.

STEP 1: Let $x_{0} \in X$ be given and set $n:=0$.

STEP 2: Calculate $x_{n+1} \in X$ such that, for all $y \in K$,

$$
\left\langle F_{\rho_{n+1}, \varepsilon_{n+1}}\left(x_{n+1}\right), y-x_{n+1}\right\rangle+\varphi_{\rho_{n+1}}(y)-\varphi_{\rho_{n+1}}\left(x_{n+1}\right)+\left\langle T(y)-T\left(x_{n+1}\right), x_{n+1}-x_{n}\right\rangle \notin-\operatorname{int}(C) .
$$

STEP 3: Set $n:=n+1$ and go to Step 2 .

Theorem 4.2. Suppose that $X$ is a reflexive Banach space and $K$ is a nonempty, closed and convex subset of $X$. Let $F, G: K \rightarrow \mathscr{L}(X, Z)$ be two operators and $T: X \rightarrow \mathscr{L}(X, Z)$ be a bounded linear operator. Assume that there exists $\varpi \in \operatorname{int}\left(C^{*}\right)$ such that $\langle\varpi, z\rangle \geq 0$ for all $z \notin-\operatorname{int}(C)$. Suppose that the following conditions hold:

(i) $F$ is $C$-monotone and $C$-upper hemicontinuous;

(ii) $G$ is $\boldsymbol{b}$-C-strongly monotone and $C$-upper hemicontinuous;

(iii) $\varphi$ is $C$-convex and $C$-lower semicontinuous;

(iv) there exists $\boldsymbol{m} \in \operatorname{int}(C)$ such that $\langle G(x), y-x\rangle+\|x-y\|^{2} \boldsymbol{m} \in C$ for all $x, y \in K$;

(v) $T$ is a-C-strongly positive with modulus $\boldsymbol{a} \in \operatorname{int}(C)$.

Moreover, suppose that the following conditions are satisfied:

$\left(\mathrm{H}_{1}\right)$ there exists $k \in(0,1)$ such that $\frac{\rho_{n+1}}{\rho_{n}} \frac{\left\|T_{s}\right\|}{\left[\sigma_{3}+\rho_{n+1}\left(\sigma_{1} \varepsilon_{n}-\sigma_{2}\left(\varepsilon_{n}-\varepsilon_{n+1}\right)\right)\right]}<k$, where $\sigma_{1}=\langle\varpi, \boldsymbol{b}\rangle, \sigma_{2}=\langle\varpi, \boldsymbol{m}\rangle$ and $\sigma_{3}=\langle\varpi, \boldsymbol{a}\rangle$;

$\left(\mathrm{H}_{2}\right)$ the sequence $\alpha_{n}:=\left[\rho_{n} \varepsilon_{n}\right]^{-1}$ is bounded.

Then, the iterative sequence generated by Algorithm 4.1 converges strongly to a solution $\bar{x}$ of (2.2).

Proof. From Algorithm 4.1, we have

$$
\begin{array}{r}
\rho_{n+1}\left[\left\langle F\left(x_{n+1}\right)+\varepsilon_{n+1} G\left(x_{n+1}\right), y-x_{n+1}\right\rangle+\varphi(y)-\varphi\left(x_{n+1}\right)\right] \\
+\left\langle T(y)-T\left(x_{n+1}\right), x_{n+1}-x_{n}\right\rangle \notin-\operatorname{int}(C), \quad \text { for all } y \in K .
\end{array}
$$

and

$$
\begin{array}{r}
\rho_{n}\left[\left\langle F\left(x_{n}\right)+\varepsilon_{n} G\left(x_{n}\right), y-x_{n}\right\rangle+\varphi(y)-\varphi\left(x_{n}\right)\right]+\left\langle T(y)-T\left(x_{n}\right), x_{n}-x_{n-1}\right\rangle \notin-\operatorname{int}(C), \\
\text { for all } y \in K .
\end{array}
$$

By taking $y=x_{n}$ in (4.17) and $y=x_{n+1}$ in (4.18), we get

$$
\begin{gathered}
{\left[\left\langle F\left(x_{n+1}\right)+\varepsilon_{n+1} G\left(x_{n+1}\right), x_{n}-x_{n+1}\right\rangle+\varphi\left(x_{n}\right)-\varphi\left(x_{n+1}\right)\right]} \\
\quad+\frac{1}{\rho_{n+1}}\left\langle T\left(x_{n}\right)-T\left(x_{n+1}\right), x_{n+1}-x_{n}\right\rangle \notin-\operatorname{int}(C),
\end{gathered}
$$

and

$$
\begin{gathered}
{\left[\left\langle F\left(x_{n}\right)+\varepsilon_{n} G\left(x_{n}\right), x_{n+1}-x_{n}\right\rangle+\varphi\left(x_{n+1}\right)-\varphi\left(x_{n}\right)\right]} \\
+\frac{1}{\rho_{n}}\left\langle T\left(x_{n+1}\right)-T\left(x_{n}\right), x_{n}-x_{n-1}\right\rangle \notin-\operatorname{int}(C) .
\end{gathered}
$$


It follows that

$$
\begin{array}{r}
\left\langle\varpi,\left\langle F\left(x_{n+1}\right)+\varepsilon_{n+1} G\left(x_{n+1}\right), x_{n}-x_{n+1}\right\rangle+\varphi\left(x_{n}\right)-\varphi\left(x_{n+1}\right)\right\rangle \\
+\frac{1}{\rho_{n+1}}\left\langle\varpi,\left\langle T\left(x_{n}\right)-T\left(x_{n+1}\right), x_{n+1}-x_{n}\right\rangle\right\rangle \geq 0
\end{array}
$$

and

$$
\begin{array}{r}
\left\langle\varpi,\left\langle F\left(x_{n}\right)+\varepsilon_{n} G\left(x_{n}\right), x_{n+1}-x_{n}\right\rangle+\varphi\left(x_{n+1}\right)-\varphi\left(x_{n}\right)\right\rangle \\
+\frac{1}{\rho_{n}}\left\langle\varpi,\left\langle T\left(x_{n+1}\right)-T\left(x_{n}\right), x_{n}-x_{n-1}\right\rangle\right\rangle \geq 0
\end{array}
$$

By adding (4.19) and (4.20), we obtain

$$
\begin{array}{r}
\left\langle\varpi,\left\langle F\left(x_{n+1}\right)-F\left(x_{n}\right), x_{n}-x_{n+1}\right\rangle\right\rangle+\varepsilon_{n}\left\langle\varpi,\left\langle G\left(x_{n+1}\right)-G\left(x_{n}\right), x_{n}-x_{n+1}\right\rangle\right\rangle \\
+\left(\varepsilon_{n+1}-\varepsilon_{n}\right)\left\langle\varpi,\left\langle G\left(x_{n+1}\right), x_{n}-x_{n+1}\right\rangle\right\rangle+\frac{1}{\rho_{n+1}}\left\langle\varpi,\left\langle T\left(x_{n}-x_{n+1}\right), x_{n+1}-x_{n}\right\rangle\right\rangle \\
+\frac{1}{\rho_{n}}\left\langle\varpi,\left\langle T\left(x_{n+1}\right)-T\left(x_{n}\right), x_{n}-x_{n-1}\right\rangle\right\rangle \geq 0 .
\end{array}
$$

Since $F$ is $C$-monotone and $G$ is $\mathbf{b}-C$-strongly monotone, we derive from (4.21) that

$$
\begin{aligned}
& \left(\varepsilon_{n+1}-\varepsilon_{n}\right)\left\langle\varpi,\left\langle G\left(x_{n+1}\right), x_{n}-x_{n+1}\right\rangle\right\rangle-\varepsilon_{n}\langle\varpi, \mathbf{b}\rangle\left\|x_{n+1}-x_{n}\right\|^{2} \\
& \quad+\frac{1}{\rho_{n+1}}\left\langle\varpi,\left\langle T\left(x_{n}-x_{n+1}\right), x_{n+1}-x_{n}\right\rangle\right\rangle+\frac{1}{\rho_{n}}\left\langle\varpi,\left\langle T\left(x_{n+1}\right)-T\left(x_{n}\right), x_{n}-x_{n-1}\right\rangle\right\rangle \geq 0 .
\end{aligned}
$$

In view of condition (iv), we deduce from (4.22) that

$$
\begin{aligned}
& \left(\varepsilon_{n}-\varepsilon_{n+1}\right)\langle\varpi, \mathbf{m}\rangle\left\|x_{n+1}-x_{n}\right\|^{2}-\varepsilon_{n}\langle\varpi, \mathbf{b}\rangle\left\|x_{n+1}-x_{n}\right\|^{2} \\
& \quad+\frac{1}{\rho_{n+1}}\left\langle\varpi,\left\langle T\left(x_{n}-x_{n+1}\right), x_{n+1}-x_{n}\right\rangle\right\rangle+\frac{1}{\rho_{n}}\left\langle\varpi,\left\langle T\left(x_{n+1}\right)-T\left(x_{n}\right), x_{n}-x_{n-1}\right\rangle\right\rangle \geq 0 .
\end{aligned}
$$

Let us set $\sigma_{1}:=\langle\varpi, \mathbf{b}\rangle$ and $\sigma_{2}:=\langle\varpi, \mathbf{m}\rangle$ which are strictly positive since $\varpi \in \operatorname{int}\left(C^{*}\right)$ and $\mathbf{b}, \mathbf{m} \in \operatorname{int}(C)$. Therefore, from (4.22), we have

$$
\begin{aligned}
\left\|x_{n+1}-x_{n}\right\|^{2} \leq & \frac{1}{\rho_{n+1}}\left\langle\varpi,\left\langle T\left(x_{n}-x_{n+1}\right), x_{n+1}-x_{n}\right\rangle\right\rangle \\
& +\frac{1}{\rho_{n}}\left\langle\varpi,\left\langle T\left(x_{n+1}\right)-T\left(x_{n}\right), x_{n}-x_{n-1}\right\rangle\right\rangle .
\end{aligned}
$$

On the other hand, since $T$ is a- $C$-strongly positive, we have

$$
\left\langle\varpi,\left\langle T\left(x_{n+1}-x_{n}\right), x_{n+1}-x_{n}\right\rangle\right\rangle \geq\langle\varpi, \mathbf{a}\rangle\left\|x_{n+1}-x_{n}\right\|^{2} .
$$

Hence, by setting $\sigma_{3}:=\langle\varpi, \mathbf{a}\rangle$ which is strictly positive, we deduce from (4.24) that

$$
\left[\frac{\sigma_{3}}{\rho_{n+1}}+\sigma_{1} \varepsilon_{n}-\sigma_{2}\left(\varepsilon_{n}-\varepsilon_{n+1}\right)\right]\left\|x_{n+1}-x_{n}\right\|^{2} \leq \frac{1}{\rho_{n}}\left\langle\varpi,\left\langle T\left(x_{n+1}-x_{n}\right), x_{n}-x_{n-1}\right\rangle\right\rangle .
$$

Therefore,

$$
\left[\frac{\sigma_{3}}{\rho_{n+1}}+\sigma_{1} \varepsilon_{n}-\sigma_{2}\left(\varepsilon_{n}-\varepsilon_{n+1}\right)\right]\left\|x_{n+1}-x_{n}\right\|^{2} \leq \frac{1}{\rho_{n}}\left\|T_{\varpi}\right\|\left\|x_{n+1}-x_{n}\right\|\left\|x_{n}-x_{n-1}\right\| .
$$


Consequently, we obtain

$$
\left\|x_{n+1}-x_{n}\right\| \leq \frac{\rho_{n+1}}{\rho_{n}} \frac{\left\|T_{\varpi}\right\|}{\left[\sigma_{3}+\rho_{n+1}\left(\sigma_{1} \varepsilon_{n}-\sigma_{2}\left(\varepsilon_{n}-\varepsilon_{n+1}\right)\right)\right]}\left\|x_{n}-x_{n-1}\right\| .
$$

Hence, by using condition $\left(\mathrm{H}_{1}\right)$, we deduce that

$$
\left\|x_{n+1}-x_{n}\right\| \leq k\left\|x_{n}-x_{n-1}\right\| \text { with } k<1 .
$$

Thus, $x_{n}$ converges strongly to a point $\bar{x} \in K$.

Now, let us show that $\bar{x}$ is a solution of the bilevel problem (2.2). To this aim, from Algorithm 4.1, we have that, for all $n \in \mathbb{N}$ and all $y \in K$,

$$
\left\langle F_{\rho_{n+1}, \varepsilon_{n+1}}\left(x_{n+1}\right), y-x_{n+1}\right\rangle+\varphi_{\rho_{n+1}}(y)-\varphi_{\rho_{n+1}}\left(x_{n+1}\right)+\left\langle T(y)-T\left(x_{n+1}\right), x_{n+1}-x_{n}\right\rangle \notin-\operatorname{int}(C) .
$$

As $F_{\rho_{n+1}, \varepsilon_{n+1}}$ is $C$-monotone, it follows that

$$
\left\langle F_{\rho_{n+1}, \varepsilon_{n+1}}(y)-F_{\rho_{n+1}, \varepsilon_{n+1}}\left(x_{n+1}\right), y-x_{n+1}\right\rangle \in C, \quad \text { for all } y \in K .
$$

From (4.25), (4.26) and Lemma 2.1, we obtain, for all $y \in K$

$$
\left\langle F_{\rho_{n+1}, \varepsilon_{n+1}}(y), y-x_{n+1}\right\rangle+\varphi_{\rho_{n+1}}(y)-\varphi_{\rho_{n+1}}\left(x_{n+1}\right)+\left\langle T(y)-T\left(x_{n+1}\right), x_{n+1}-x_{n}\right\rangle \notin-\operatorname{int}(C) .
$$

Hence, we have, for all $y \in K$,

$$
\begin{aligned}
\left\langle\varpi,\left\langle F(y), x_{n+1}-y\right\rangle+\varphi\left(x_{n+1}\right)-\right. & \varphi(y)\rangle+\varepsilon_{n+1}\left\langle\varpi,\left\langle G(y), x_{n+1}-y\right\rangle\right\rangle \\
& \leq \frac{1}{\rho_{n+1}}\left\langle T_{\varpi}\left(y-x_{n+1}\right), x_{n+1}-x_{n}\right\rangle .
\end{aligned}
$$

By considering the limit when $n \rightarrow+\infty$ in (4.27), we obtain

$$
\langle\varpi,\langle F(y), \bar{x}-y\rangle+\varphi(\bar{x})-\varphi(y)\rangle \leq 0, \quad \text { for all } y \in K .
$$

Since $\varpi \in \operatorname{int}\left(C^{*}\right)$ and $\operatorname{int}\left(C^{*}\right)=\left\{\lambda \in Z^{*}:\langle\lambda, z\rangle>0\right.$ for all $\left.z \in \operatorname{int}(C)\right\}$, it follows that

$$
\langle F(y), y-\bar{x}\rangle+\varphi(y)-\varphi(\bar{x})\rangle \notin-\operatorname{int}(C), \quad \text { for all } y \in K .
$$

For $t \in(0,1)$ and $y \in K$, let us set $y_{t}:=\bar{x}+t(y-\bar{x}) \in K$. As $\varphi$ is $C$-convex, it follows that

$$
t \varphi(y)+(1-t) \varphi(\bar{x})-\varphi\left(y_{t}\right) \in C .
$$

Therefore, from relation (4.28) considered for $y_{t}$, relation (4.29) and Lemma 2.1, we obtain

$$
\left.\left\langle F\left(y_{t}\right), y-\bar{x}\right\rangle+\varphi(y)-\varphi(\bar{x})\right\rangle \notin-\operatorname{int}(C) .
$$

As $F$ is $C$-upper hemicontinuous, we deduce from (4.30) that

$$
\langle F(\bar{x}), y-\bar{x}\rangle+\varphi(y)-\varphi(\bar{x})\rangle \notin-\operatorname{int}(C), \quad \text { for all } y \in K .
$$

Hence, $\bar{x}$ is a solution of the vector variational inequality problem (2.1), i.e. $\bar{x} \in \mathbb{S}$.

Now, let us verify that $\langle G(\bar{x}), z-\bar{x}\rangle \notin \operatorname{int}(C)$ for all $z \in \mathbb{S}$. From Algorithm 4.1, we have, for all $y \in K$,

$$
\left\langle F_{\rho_{n+1}, \varepsilon_{n+1}}\left(x_{n+1}\right), y-x_{n+1}\right\rangle+\varphi_{\rho_{n+1}}(y)-\varphi_{\rho_{n+1}}\left(x_{n+1}\right)+\left\langle T(y)-T\left(x_{n+1}\right), x_{n+1}-x_{n}\right\rangle \notin-\operatorname{int}(C) .
$$

Let us take $z \in \mathbb{S}$. By using the $C$-monotonicity of $F_{\rho_{n+1}, \varepsilon_{n+1}}$, we deduce from (4.31) that

$$
\left\langle F_{\rho_{n+1}, \varepsilon_{n+1}}(z), z-x_{n+1}\right\rangle+\varphi_{\rho_{n+1}}(z)-\varphi_{\rho_{n+1}}\left(x_{n+1}\right)+\left\langle T(z)-T\left(x_{n+1}\right), x_{n+1}-x_{n}\right\rangle \notin-\operatorname{int}(C) .
$$


Hence,

$$
\left\langle\varpi,\left\langle F_{\rho_{n+1}, \varepsilon_{n+1}}(z), z-x_{n+1}\right\rangle+\varphi_{\rho_{n+1}}(z)-\varphi_{\rho_{n+1}}\left(x_{n+1}\right)+\left\langle T(z)-T\left(x_{n+1}\right), x_{n+1}-x_{n}\right\rangle\right\rangle \geq 0 .
$$

It follows that

$$
\begin{array}{r}
\rho_{n+1}\left[\left\langle\varpi,\left\langle F(z), x_{n+1}-z\right\rangle+\varphi\left(x_{n+1}\right)-\varphi(z)\right\rangle\right]+\rho_{n+1} \varepsilon_{n+1}\left\langle\varpi,\left\langle G(z), x_{n+1}-z\right\rangle\right\rangle \\
\leq\left\langle\varpi,\left\langle T(z)-T\left(x_{n+1}\right), x_{n+1}-x_{n}\right\rangle\right\rangle .
\end{array}
$$

As $z \in \mathbb{S}$, we have $\left\langle\varpi,\left\langle F(z), x_{n+1}-z\right\rangle+\varphi\left(x_{n+1}\right)-\varphi(z)\right\rangle \geq 0$. Therefore, from (4.32), we obtain

$$
\left\langle\varpi,\left\langle G(z), x_{n+1}-z\right\rangle\right\rangle \leq \frac{1}{\rho_{n+1} \varepsilon_{n+1}}\left\langle\varpi,\left\langle T(z)-T\left(x_{n+1}\right), x_{n+1}-x_{n}\right\rangle\right\rangle .
$$

By considering the limit when $n \rightarrow+\infty$ in (4.33) and by taking account of condition $\left(\mathrm{H}_{2}\right)$, it follows that

$$
\langle\varpi,\langle G(z), \bar{x}-z\rangle\rangle \leq 0 .
$$

Hence, $\langle G(z), \bar{x}-z\rangle \notin \operatorname{int}(C)$. As $G$ is $C$-upper hemicontinuous, we can show, by proceeding similarly as before, that

$$
\langle G(\bar{x}), z-\bar{x}\rangle \notin-\operatorname{int}(C), \quad \text { for all } z \in \mathbb{S} .
$$

This completes the proof.

Remark 4.3. The conditions $\left(\mathrm{H}_{1}\right)$ and $\left(\mathrm{H}_{2}\right)$ hold if we consider, for instance, $\varepsilon_{n}=\frac{1}{n^{\beta}}(\beta \geq 1)$ and $\rho_{n}=\frac{1}{\varepsilon_{n}^{2}}$.

\section{Acknowledgements}

The authors are grateful to the Mohapatra Family Foundation for its Financial support through a grant to the University of Central Florida, Orlando.

\section{REFERENCES}

[1] J.F. Bard, Practical Bilevel Optimization: Applications and Algorithms, Kluwer Academic Publishers, Dordrecht, 1998.

[2] H. Bonnel, J. Morgan, Semivectorial bilevel optimization problem: Penalty approach, J. Optim. Theory Appl. 131 (2006), 365-382.

[3] S. Dempe, Annotated Bibliography on Bilevel Programming and Mathematical Programs with Equilibrium Constraints, Optimization 52 (2003), 333-359.

[4] S. Dempe, Foundations of Bilevel Programming, Kluwer Academie Publishers, Dordrecht, 2002.

[5] K. Fan, A generalization of Tychonoff's fixed point theorem, Math. Ann. 142 (1961), 305-310.

[6] F. Giannessi, Theorems of alterative, quadratic programs and complementary problems. In: R.W. Cottle, F. Giannessi, J.C. Lions, (eds.) Variational Inequality and Complementary Problems, Wiley, New York, 1980.

[7] P.T. Harker, J.S. Pang, Existence of optimal solutions to mathematcal programs with equilibrium constraints. Oper. Res. Lett. 7 (1988), 61-64.

[8] S. Komlosi, Generalised monotonicity and generalized convexity, J. Optim. Theory Appl. 84 (1995), 361-376.

[9] X. Ju, X. Zhu, M. Akram, Levitin-Polyak well-posedness for bilevel vector variational inequalities, J. Nonlinear Var. Anal. 3 (2019), 277-293.

[10] G. Kassay, M. Miholca, Existence results for vector equilibrium problems given by a sum of two functions, J. Global Optim. 63 (2015), 195-211. 
[11] C.S. Lalitha, M. Mehta, Vector variational inequalities with cone-pseudomonotone bifunctions, Optimization 54 (2005), 327-338.

[12] Y.C. Liou, X.Q. Yang, J.C. Yao, Mathematical programs with vector optimization constraints, J. Optim. Theory Appl. 162 (2005), 345-355.

[13] D.T. Luc, Theory of Vector Optimization, Lecture Notes in Economics and Mathematical Systems, vol. 319, Springer-Verlag, Berlin, 1989.

[14] Z.Q. Luo, J.S. Pang, D. Ralph, Mathematical Programs with Equilibrium Constraints, Cambridge University Press, New York, 1996.

[15] J. Outrata, M. Kocvara, J. Zowe, Nonsmooth Approach to Optimizations with Equilibrium Constraints, Kluwer Academic Publishers, Dordrecht, 1998.

[16] T. Tanaka, Generalized semicontinuity and existence theorems for cone saddle points, Appl. Math. Optim. 36 (1997), 313-322.

[17] S.J. Yu, J.C. Yao, On Vector Variational Inequalities, J. Optim. Theory Appl. 89 (1996), 749-769. 